REVISIONES

Rev Obstet Ginecol Venez. 2021; 81 (1): 49-60

https://doi.org/10.51288/00810109

\title{
La vulnerabilidad del embarazo en las trabajadoras del sexo
}

\author{
Mayara Lorenzo Maggi, ${ }^{1}$ (DD Gustavo Gonçalves dos Santos, ${ }^{2}$ (D) Francisco Romero Cabral. ${ }^{3}$
}

\begin{abstract}
RESUMEN
Objetivo: Evaluar la exposición del binomio madre-bebé a las complicaciones de las trabajadoras sexuales. Métodos: Esta es una revisión integral de la literatura que siguió el sistema requerido de 6 etapas. La búsqueda se realizó en la Biblioteca Virtual de Salud en las siguientes bases de datos: Base de datos de Enfermería (BDENF-Enfermería), Sistema de Análisis y Recuperación de Literatura Médica en Línea (Medline), Literatura Latinoamericana de Ciencias de la Salud (LiLACS), Índice Bibliográfico Español de Ciencias de la Salud (IBECS-ES), Centro Nacional de Información Biotecnológica (NCBI), Revistas SAGE, con los descriptores: profesionales del sexo e infecciones de transmisión sexual, prostitución y vulnerabilidad, de marzo a abril de 2019. Se identificaron un total de 105 artículos y, tras los criterios de exclusión e inclusión, se obtuvieron 25 publicaciones que componían la muestra.

Resultados: Basándose en el análisis de los artículos encontrados, se extrajeron las siguientes categorías: infecciones de transmisión sexual, violencia y drogas ilícitas o lícitas que están interconectadas.

Conclusiones: A pesar de la limitación de la muestra, el estudio permitió evaluar que el binomio madre-bebé es vulnerable a varias complicaciones como: aborto, restricción del crecimiento, parto prematuro, muerte fetal y/o materna, prematuridad, anormalidades fisicas y de comportamiento en la madre y bebé y desprendimiento de placenta debido a la violencia sufrida, infecciones de transmisión sexual adquiridas y sustancias utilizadas.
\end{abstract}

Palabras clave: Trabajadoras del sexo, Embarazo, Vulnerabilidad.

\section{SUMMARY}

Objective: To evaluate the exposure of the mother-baby binomial to complications in sex workers.

Methods: This is an integrative literature review that followed a 6-phase system. The research took place in the Virtual Health Library in the following databases: Nursing Database (BDENF- Nursing), Online Medical Literature Analysis and Recovery System (Medline), Latin American Literature of Health Sciences (LiLACS), Spanish Bibliographic Index of Health Sciences (IBECS-ES), National Center for Biotechnological Information (NCBI), SAGE Journals with the descriptors: sex workers and sexually transmitted infections, prostitution and sexually transmitted infections, prostitution and vulnerability, from March to April 2019; 105 articles were identified and were submitted to the exclusion and inclusion criteria, and 25 publications were obtained for the sample.

Results: Based on the analysis of the articles the following categories were determined: sexually transmitted infections (STIS), violence and illicit or licit drugs are linked.

Conclusion: Despite the limitation of sampling, the study allowed us to evaluate that the mother-baby binomial is vulnerable to several complications such as spontaneous abortion, growth restriction, premature labor, fetal and/or maternal death, prematurity, physical and behavioral abnormalities of mother and baby, and placental detachment, due to suffered violence, acquired sexually transmitted infections and used substances.

Keywords: Sex workers, Pregnancy, Vulnerability.

\begin{abstract}
'Enfermera Obstetra. Enfermera del Hospital Regional de Cotia. São Paulo - SP, Brazil. 2 Enfermero Obstetra e Profesor. Profesor del curso de Enfermería en Universidade de Santo Amaro - UNISA. Mestrando del Programa de Postgrado de la Facultad de Medicina de Botucatu da Universidade Estadual Paulista "Júlio de Mesquita Filho" FMB/UNESP. São Paulo - SP, Brazil. 3 Doctor en Neurología y Neurociencias. Profesor visitante del Programa de Posgrado en Bioquímica y Bioprospección de la Universidade Federal de Pelotas, Rio Grande do Sul, Brazil. Profesor visitante del Programa de Posgrado Stricto Sensu en Ciencias de la Salud de la Facultade de Ciências Médicas da Santa Casa de São Paulo. São Paulo - SP, Brazil.
\end{abstract}

\section{INTRODUCCIÓN}

Considerada una de las profesiones más antiguas de la humanidad, la prostitución nunca ha recibido los mismos derechos sociales, políticos y jurídicos que las demás categorías, siendo siempre estigmatizada y relacionada con la deshonestidad, la degradación y la falta de carácter (1). 
A mediados del siglo $\mathrm{XX}$, su panorama comenzó a cambiar, debido a los movimientos sociales que se manifestaron en la defensa de los derechos de las prostitutas y a la propuesta de resignificación de la prostitución como profesión (2). Pero fue solo a principios de la década de 2000 que el Ministerio de Trabajo y Empleo (MTE) reconoció a la profesional del sexo (PS) como trabajadora, componiendo la Clasificación Brasileña de Ocupación (CBO) (3).

A pesar de estos logros, en el imaginario popular, la prostitución es considerada la antítesis de la maternidad, relacionándola siempre con el aborto y el abandono, debido a la santificación creada sobre el embarazo. Sin embargo, las trabajadoras del sexo tienen una vida más allá del trabajo, y sus embarazos no siempre son indeseados, siendo fruto de relaciones amorosas duraderas, matrimonios o uniones estables (4).

Desde los albores de la humanidad, la prostitución ha estado ligada a la degradación, la deshonestidad y la falta de autonomía. Estas mujeres están constantemente expuestas a diversos factores de riesgo, como la sumisión y, sobre todo, el uso abusivo de bebidas alcohólicas y drogas ilícitas. También experimentan un escenario rodeado de agresiones, abusos, humillaciones y ofensas, incluyéndolas en el grupo de personas vulnerables $(1,2)$.

En la prostitución, hay varios actores, pero dos reciben mayor protagonismo: la prostituta y el cliente. El intercambio de sexo por dinero está directamente relacionado con las necesidades del mundo capitalista. Estas necesidades no solo están ligadas a la supervivencia, sino también al bienestar, el estatus y la aceptación social. En la sociedad actual, donde las personas valen lo que tienen, genera estatus y representa el poder $(1,2,4)$.

Cuando se arregla esta etiqueta, se olvida que estas mujeres prostitutas tienen otros roles en sus vidas. La prostitución es su trabajo, su forma de mantenerse y ganar dinero, pero, fuera de eso, ocupan el papel de madre, hija, esposa, es decir, gente común. La prostitución no forma parte de la personalidad del sujeto, pero sí de su vida. Así como el psicólogo no nació profesionalmente y solo ejerce su profesión en su lugar de trabajo, empresas, clubes, escuelas, oficinas, la prostituta no nació con esta característica y se desconecta cuando no está trabajando (4 - 6).

El MTE entiende que la prostitución es vista como el intercambio de sexo por dinero, sin ningún vínculo afectivo entre quienes la realizan. Consideradas por el MTE como trabajadoras del sexo, estas mujeres siguen siendo discriminadas, marginadas y lidian con diferentes tipos de prejuicios. Es importante distinguir a las mujeres que entran en el meretricio para intentar mejorar sus ingresos, de las que necesitan prostituirse como medio de subsistencia, porque las realidades socioeconómicas son muy diferentes, aunque el objetivo en ambos casos es el beneficio económico. Cabe señalar que la mayoría de las trabajadoras del sexo son mujeres de bajos ingresos que están expuestas a condiciones de vida y de trabajo insalubres y precarias $(4-6)$.

Debido al valor moral que simboliza la familia para las prostitutas, estas mujeres suelen estar sometidas a diversos factores de riesgo, que pueden comprometer el desarrollo del embarazo. Frente a este análisis, el propósito de esta investigación es evaluar las posibles complicaciones a las que se expone el binomio madrebebé debido a la actividad realizada.

\section{MÉTODOS}

Se trata de una revisión interactiva de la literatura, que incluye el análisis de las investigaciones pertinentes que respaldan la adopción de decisiones, lo que permite la incorporación de pruebas en la práctica. Este tipo de estudio tiene el propósito de sintetizar un tema, además de señalar las lagunas que deben ser llenadas con la ejecución de nuevas investigaciones 
(5). La construcción se realizó de acuerdo a las seis fases que requiere el método: definición del sujeto y elaboración de la pregunta guía, muestreo o búsqueda de literatura, recolección de datos, análisis crítico de los estudios incluidos, discusión e interpretación de los resultados y presentación de la revisión (6).

Definición del tema y elaboración de la pregunta orientadora

La pregunta guía para la revisión fue: ¿Cuáles son los posibles factores de riesgo a los que pueden enfrentarse las profesionales del sexo durante el embarazo? Así, se esbozó el siguiente objetivo: evaluar la exposición del binomio madre-bebé a las complicaciones en las profesionales del sexo.

\section{Búsqueda en la literatura}

La búsqueda se realizó en la Biblioteca Virtual de Salud, en las siguientes bases de datos: Base de datos de Enfermería (BDENF- Enfermería), Sistema de Análisis y Recuperación de Literatura Médica en Línea (Medline), Literatura Latinoamericana de Ciencias de la Salud (LiLACS), Índice Bibliográfico Español de Ciencias de la Salud (IBECS-ES), Centro Nacional de Información Biotecnológica (NCBI), Revistas SAGE, en el período del 06/03/19 al 27/04/19, con los siguientes descriptores: trabajadoras sexuales e infecciones de transmisión sexual (ITS), prostitución y vulnerabilidad.

\section{Criterios de inclusión y exclusión}

Se incluyeron los artículos que cumplían el objetivo del estudio, disponibles en su totalidad, en inglés, español o portugués, de los últimos cinco años, y los criterios de exclusión fueron los que se repitieron y que no eran pertinentes para la investigación.

\section{Análisis crítico de los estudios incluidos}

Se adoptó la estrategia de selección de la lectura del título de la publicación, seguida de una lectura cautelosa del resumen; en los casos en que no eran suficientes para definir el tema, se buscaba el texto en su totalidad tratando de asociarlo con el objetivo.

Debate, interpretación de los resultados y presentación de la revisión

Se encontró un total de 105 publicaciones mediante la búsqueda, pero el número total se redujo a 24 (22,8 \%) que cumplían los criterios de inclusión, con los datos reunidos organizados en un cuadro. Después de la tabulación, se hizo un análisis detallado que presentaba los resultados de manera descriptiva. Los datos incluidos en este estudio se analizaron mediante estadísticas descriptivas, a partir de los pasajes que configuran la evidencia científica, dividiéndolos por las similitudes temáticas encontradas en la literatura. Los resultados de los estudios fueron interpretados y emanaron en una evaluación crítica y síntesis de la evidencia disponible del tema investigado.

\section{RESULTADOS}

Con las estrategias de búsqueda se encontraron 105 estudios. De ellos, 24 respondieron a la pregunta orientadora y a los criterios de inclusión. En el cuadro 1 se muestran los artículos seleccionados de L1 a L24 (7-30), según su título, autor, año, objetivo y conclusión. La mayoría de las publicaciones (41,6\%) datan de 2014, seguidas de 2017 (33,3 \%), y no hubo publicaciones en el año 2018, como se puede ver en el cuadro 1 . En cuanto al diseño y el idioma del estudio, la mayor prevalencia $(37,5 \%)$ fue transversal y el idioma inglés $(58,3 \%)$. El cuadro 1 muestra una síntesis general del objetivo y la conclusión encontrados en los estudios para mejorar la comprensión del debate. 
Cuadro 1. Presentación del estudio sobre el objetivo y la conclusión

\begin{tabular}{|c|c|c|c|}
\hline Cód & TÍTULO & OBJETIVO & CONCLUSIÓN \\
\hline L 1 & $\begin{array}{l}\text { Prevalence and predictors } \\
\text { of sexual violence among } \\
\text { commercial sex workers in } \\
\text { Northern Ethiopia ( } 7 \text { ). }\end{array}$ & $\begin{array}{l}\text { Investigar la prevalencia, el carácter de la } \\
\text { violencia sexual y los factores de riesgo } \\
\text { que experimentan los profesionales del } \\
\text { sexo. }\end{array}$ & $\begin{array}{l}\text { La prevalencia de la violencia era } \\
\text { alta, siendo el consumo de drogas, los } \\
\text { bajos ingresos y la baja escolaridad los } \\
\text { principales factores de riesgo. }\end{array}$ \\
\hline L 2 & $\begin{array}{l}\text { A assistência à saúde } \\
\text { das profissionais do sexo } \\
\text { no } \text { Brasil: uma revisão } \\
\text { integrativa (8). }\end{array}$ & $\begin{array}{l}\text { Saber cómo la literatura aborda el } \\
\text { cuidado de la salud de las mujeres en } \\
\text { situación de prostitución. }\end{array}$ & $\begin{array}{l}\text { Se ha comprobado que hay pocos } \\
\text { estudios que aborden indirectamente } \\
\text { las cuestiones relacionadas con las } \\
\text { profesionales del sexo. }\end{array}$ \\
\hline L 3 & $\begin{array}{l}\text { Prostituição, HIV/Aids e } \\
\text { vulnerabilidades: a "cama da } \\
\text { casa" e a "cama da rua" (9). }\end{array}$ & $\begin{array}{l}\text { Analizar entre las trabajadoras del } \\
\text { sexo cómo las concepciones sobre } \\
\text { la prostitución, el amor y la familia } \\
\text { han influido en la prevención de las } \\
\text { enfermedades de transmisión sexual, } \\
\text { en la diferenciación entre el cliente en } \\
\text { la prostitución y la pareja fija en la vida } \\
\text { personal. }\end{array}$ & $\begin{array}{l}\text { El riesgo de infección por VIH en la calle } \\
\text { se reiteró con la constante declaración } \\
\text { sobre el uso de preservativos en la } \\
\text { prostitución. Identificando, que también } \\
\text { hay riesgos inherentes a las prácticas } \\
\text { sexuales en el trabajo, que demandan } \\
\text { estrategias de protección más allá del } \\
\text { uso del condón. }\end{array}$ \\
\hline L 4 & $\begin{array}{l}\text { Trabajadores del sexo y salud } \\
\text { pública: intersecciones, } \\
\text { vulnerabilidades } \\
\text { resistência (10). }\end{array}$ & $\begin{array}{l}\text { Discutir las vulnerabilidades sociales, } \\
\text { laborales y legales que afectan a los } \\
\text { trabajadores sexuales y cómo interfieren } \\
\text { con su salud. }\end{array}$ & $\begin{array}{l}\text { En los países occidentales, la tasa de } \\
\text { VIH en las trabajadoras del sexo es baja, } \\
\text { con la excepción de los consumidores } \\
\text { de drogas inyectables. Además, las } \\
\text { trabajadoras están sometidas a la } \\
\text { estigmatización y otras formas de } \\
\text { violencia. }\end{array}$ \\
\hline L 5 & $\begin{array}{l}\text { Situações de vulnerabilidade } \\
\text { à violência de mulheres } \\
\text { profissionais do sexo: } \\
\text { interfaces no campo da } \\
\text { saúde (11). }\end{array}$ & $\begin{array}{l}\text { Provocar reflexiones teóricas sobre } \\
\text { las situaciones de vulnerabilidad a la } \\
\text { violencia de las mujeres profesionales } \\
\text { del sexo y las interfaces de este problema } \\
\text { en el ámbito de la salud. }\end{array}$ & $\begin{array}{l}\text { Las reflexiones muestran la necesidad de } \\
\text { formular estrategias de salud que tengan } \\
\text { en cuenta aspectos únicos y específicos } \\
\text { de las trabajadoras del sexo. }\end{array}$ \\
\hline L 6 & $\begin{array}{l}\text { Prevalence and Correlates } \\
\text { of HIV and Sexually } \\
\text { Transmitted Infections } \\
\text { among Female Sex Workers } \\
\text { and Their Non-commercial } \\
\text { Male Partners in Two } \\
\text { Mexico-USA Border Cities } \\
(12) \text {. }\end{array}$ & $\begin{array}{l}\text { Determinar la prevalencia y los } \\
\text { correlatos del VIH/ITS en las relaciones } \\
\text { íntimas de las trabajadoras del sexo en el } \\
\text { norte de México. }\end{array}$ & $\begin{array}{l}\text { En las relaciones íntimas de las } \\
\text { trabajadoras del sexo en dos ciudades } \\
\text { fronterizas mexicanas y americanas, una } \\
\text { de cada diez parejas dio positivo en las } \\
\text { pruebas de VIH/ITS. }\end{array}$ \\
\hline L 7 & $\begin{array}{l}\text { Aspectos de vida e saúde das } \\
\text { profissionais do sexo (13). }\end{array}$ & $\begin{array}{l}\text { Analizar las condiciones de vida y la } \\
\text { salud de las trabajadoras del sexo. }\end{array}$ & $\begin{array}{l}\text { Los aspectos de la vida y la salud } \\
\text { de las profesionales del sexo se han } \\
\text { relacionado, con mayor prevalencia, } \\
\text { con la difusión de las infecciones de } \\
\text { transmisión sexual, y es necesario } \\
\text { ampliar los debates sobre su calidad de } \\
\text { vida. }\end{array}$ \\
\hline
\end{tabular}


Cuadro 1. Presentación del estudio sobre el objetivo y la conclusión (cont.)

\begin{tabular}{|c|c|c|c|}
\hline Cód & TÍTULO & OBJETIVO & CONCLUSIÓN \\
\hline L 8 & $\begin{array}{l}\text { Prevalence of sexually } \\
\text { transmitted infections and } \\
\text { their risk factors among } \\
\text { female sex workers in } \\
\text { Isfahan, Iran: A cross- } \\
\text { sectional study (14). }\end{array}$ & $\begin{array}{l}\text { Estimar la prevalencia de las ITS entre } \\
\text { los profesionales que asistieron al Centro } \\
\text { de Reducción de Daños para Mujeres en } \\
\text { Isfahan, Irán. }\end{array}$ & $\begin{array}{l}\text { El diagnóstico de las infecciones de } \\
\text { transmisión sexual es defectuoso y } \\
\text { es necesario mejorar los recursos } \\
\text { disponibles, además de controlarlas con } \\
\text { programas de intervención. }\end{array}$ \\
\hline L 9 & $\begin{array}{l}\text { Risk perception of sexually } \\
\text { transmitted infections and } \\
\text { HIV in Nigerian commercial } \\
\text { sex workers in Barcelona: a } \\
\text { qualitative study (15). }\end{array}$ & $\begin{array}{l}\text { Determinar en detalle el riesgo percibido } \\
\text { de infecciones de transmisión sexual } \\
\text { (ITS) y VIH, y las circunstancias } \\
\text { contextuales, en Nigeria de las } \\
\text { profesionales del sexo. }\end{array}$ & $\begin{array}{l}\text { Las trabajadoras del sexo pertenecen a } \\
\text { un grupo vulnerable debido a sus bajos } \\
\text { ingresos. Sus riesgos están asociados } \\
\text { con sus comportamientos como: } \\
\text { creencias, influencias y experiencias y } \\
\text { determinantes personales. }\end{array}$ \\
\hline L 10 & $\begin{array}{l}\text { Testing commercial sex } \\
\text { workers for sexually } \\
\text { transmitted infections in } \\
\text { Victoria, Australia: An } \\
\text { Evaluation of the impact of } \\
\text { reducing the frequency of } \\
\text { testing (16). }\end{array}$ & $\begin{array}{l}\text { Determinar si el cambio en la frecuencia } \\
\text { de las pruebas de detección para las } \\
\text { profesionales del sexo daría lugar a un } \\
\text { aumento del servicio a los clientes de } \\
\text { alto riesgo. }\end{array}$ & $\begin{array}{l}\text { El cambio en la frecuencia de las } \\
\text { pruebas de detección, de mensual a } \\
\text { trimestral, resultó en un aumento del } \\
15 \% \text { en los diagnósticos de ITS en la } \\
\text { clínica. En general, el cambio en la } \\
\text { frecuencia puede haber tenido un efecto } \\
\text { beneficioso en el control de las ITS en } \\
\text { Victoria. }\end{array}$ \\
\hline L 11 & $\begin{array}{l}\text { Prevalence and correlates of } \\
\text { client-perpetrated violence } \\
\text { against female sex works in } \\
13 \text { Mexican Cities (17). }\end{array}$ & $\begin{array}{l}\text { Identificar la prevalencia y los correlatos } \\
\text { de tres tipos de violencia contra las } \\
\text { trabajadoras del sexo: sexual, física y } \\
\text { económica. }\end{array}$ & $\begin{array}{l}\text { Los correlatos incluían el contexto } \\
\text { laboral, el trabajo y los factores sociales } \\
\text { y de comportamiento, lo que sugiere } \\
\text { que los enfoques de la prevención de la } \\
\text { violencia deben ser multidimensionales. }\end{array}$ \\
\hline L 12 & $\begin{array}{l}\text { A border context of violence: } \\
\text { Mexican female sex workers } \\
\text { on the U.S.-Mexico Border } \\
(18) \text {. }\end{array}$ & $\begin{array}{l}\text { Comprender la violencia diaria asociada } \\
\text { con el trabajo sexual dentro del contexto } \\
\text { único de México. }\end{array}$ & $\begin{array}{l}\text { Los demás empleados de la industria } \\
\text { deben ser regulados para garantizar } \\
\text { que no exploten a las trabajadoras del } \\
\text { sexo. El riesgo sexual se incrementa a } \\
\text { través de la subyugación violenta de los } \\
\text { clientes. }\end{array}$ \\
\hline L 13 & $\begin{array}{l}\text { Systematic review of sex } \\
\text { work intervention in sub- } \\
\text { Saharan Africa: examining } \\
\text { combination } \\
\text { approaches (19). }\end{array}$ & $\begin{array}{l}\text { Actualizar las pruebas sobre la eficacia } \\
\text { de las intervenciones de las profesionales } \\
\text { del sexo en el África subsahariana y } \\
\text { proporcionar más información sobre la } \\
\text { prevención combinada. }\end{array}$ & $\begin{array}{l}\text { Las pruebas muestran diversos modos } \\
\text { de intervención para prevenir el VIH } \\
\text { en los profesionales, y se necesitan } \\
\text { enfoques multicomponentes. }\end{array}$ \\
\hline L 14 & $\begin{array}{l}\text { Motherhood and risk for } \\
\text { Human Immunodeficiency } \\
\text { Virus/ Sexually Transmitted } \\
\text { Infections Among female } \\
\text { sex workers in the Mexico- } \\
\text { US Border Region (20). }\end{array}$ & $\begin{array}{l}\text { Examinar si la maternidad está asociada } \\
\text { con el aumento del comportamiento } \\
\text { sexual de riesgo relacionado con el VIH/ } \\
\text { ITS con clientes entre Tijuana y Ciudad } \\
\text { Juárez, México. }\end{array}$ & $\begin{array}{l}\text { Hay un atisbo de la relación entre } \\
\text { la maternidad y las mujeres que son } \\
\text { trabajadoras sexuales. Entender la } \\
\text { convergencia entre el trabajo maternal y } \\
\text { el trabajo sexual, y cómo puede influir } \\
\text { en la toma de decisiones y afectar su } \\
\text { salud es esencial para diseñar programas } \\
\text { efectivos. }\end{array}$ \\
\hline
\end{tabular}


Cuadro 1. Presentación del estudio sobre el objetivo y la conclusión (cont.)

\begin{tabular}{|c|c|c|c|}
\hline Cód & TÍTULO & OBJETIVO & CONCLUSIÓN \\
\hline L 15 & 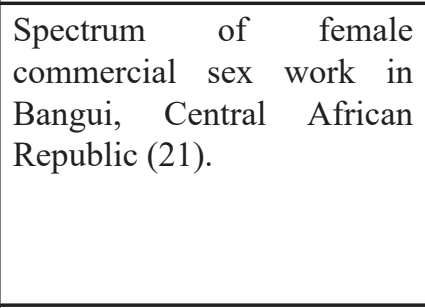 & $\begin{array}{l}\text { Clasificar a las trabajadoras del sexo } \\
\text { según criterios socioantropológicos } \\
\text { y examinar la asociación entre una } \\
\text { selección de variables demográficas y } \\
\text { de riesgo con diferentes categorías de } \\
\text { trabajo sexual femenino como resultado. }\end{array}$ & $\begin{array}{l}\text { Los resultados destacan el alto nivel } \\
\text { de vulnerabilidad de las trabajadoras } \\
\text { sexuales pobres y no profesionales } \\
\text { (kata), que se tiene especialmente en } \\
\text { cuenta a la hora de planificar programas } \\
\text { de prevención dirigidos a esta población } \\
\text { para el control de las ITS/VIH. }\end{array}$ \\
\hline L 16 & $\begin{array}{l}\text { O par e o ímpar: o potencial } \\
\text { de gestão de risco para a } \\
\text { prevenção de DST/HIV/ } \\
\text { AIDS em contextos de } \\
\text { prostituição (22). }\end{array}$ & $\begin{array}{l}\text { Elaborar recomendaciones de estrategias } \\
\text { para aumentar la autonomía de las } \\
\text { prostitutas a fin de que tomen decisiones } \\
\text { informadas para cuidar su salud, } \\
\text { promover sus derechos y evitar que } \\
\text { contraigan el VIH. }\end{array}$ & $\begin{array}{l}\text { Se necesita una acción política y una } \\
\text { investigación que incluya el entorno } \\
\text { y el contexto en el que trabajan las } \\
\text { profesionales del sexo, que reincorpore } \\
\text { el marco de derechos humanos y } \\
\text { ciudadanía que dominó la respuesta } \\
\text { brasileña hasta finales del decenio } \\
\text { de } 2000 \text {, y que las prostitutas sean } \\
\text { consideradas y tratadas como mujeres, } \\
\text { en igualdad de condiciones con todas las } \\
\text { demás. }\end{array}$ \\
\hline L 17 & $\begin{array}{l}\text { Fatores de risco para doenças } \\
\text { sexualmente transmissíveis } \\
\text { em profissionais do sexo do } \\
\text { interior piauiense (23). }\end{array}$ & $\begin{array}{l}\text { Identificar los factores de riesgo de } \\
\text { enfermedades de transmisión sexual en } \\
\text { las profesionales del sexo y verificar la } \\
\text { asociación entre el uso del preservativo } \\
\text { masculino por parte de la pareja y el } \\
\text { cliente y las características de estas } \\
\text { mujeres. }\end{array}$ & $\begin{array}{l}\text { El desarrollo de estrategias dirigidas a la } \\
\text { realidad que viven es fundamental para } \\
\text { promover su salud. }\end{array}$ \\
\hline L 18 & $\begin{array}{l}\text { Sexual and reproductive } \\
\text { health outcomes among } \\
\text { female sex workers } \\
\text { in Johannerburg and } \\
\text { Pretoria, South Africa: } \\
\text { Recommendations for public } \\
\text { health programmes (24). }\end{array}$ & $\begin{array}{l}\text { Describir las características } \\
\text { sociodemográficas y de comportamiento } \\
\text { de las profesionales del sexo en dos } \\
\text { ciudades e identificar los factores de } \\
\text { riesgo de resultados adversos en materia } \\
\text { de salud sexual y reproductiva (SSR). }\end{array}$ & $\begin{array}{l}\text { Las poblaciones de las trabajadoras del } \\
\text { sexo son heterogéneas. La segmentación } \\
\text { de las poblaciones según la edad, el país } \\
\text { de origen y el lugar de prestación de } \\
\text { servicios y el empoderamiento de los } \\
\text { proveedores de atención de la salud puede } \\
\text { ayudar a prevenir nuevas infecciones } \\
\text { por el VIH, mejorar la observancia del } \\
\text { tratamiento antirretroviral y aumentar } \\
\text { los servicios de SSR. }\end{array}$ \\
\hline L 19 & $\begin{array}{l}\text { Health education with } \\
\text { prostitutes: an experience to } \\
\text { couple of education (25). }\end{array}$ & $\begin{array}{l}\text { Identificar la experiencia de los } \\
\text { educadores sociales sobre las actividades } \\
\text { de educación sanitaria con prostitutas. }\end{array}$ & $\begin{array}{l}\text { Se necesitan programas de intervención } \\
\text { más eficaces para facilitar la labor } \\
\text { educativa, así como la creación de } \\
\text { capacidad para la educación entre pares. }\end{array}$ \\
\hline L 20 & $\begin{array}{l}\text { HIV risks var according } \\
\text { to type of sex work in a } \\
\text { cross-sectional survery from } \\
\text { Nagaland, India (26). }\end{array}$ & $\begin{array}{l}\text { Investigar la relación entre las categorías } \\
\text { de trabajo sexual y los riesgos de VIH }\end{array}$ & $\begin{array}{l}\text { Los resultados indican que las diferentes } \\
\text { categorías de trabajo sexual están } \\
\text { asociadas a diferentes perfiles de riesgo } \\
\text { de VIH e ITS }\end{array}$ \\
\hline
\end{tabular}


Cuadro 1. Presentación del estudio sobre el objetivo y la conclusión (cont.)

\begin{tabular}{|c|c|c|c|}
\hline Cód & TÍTULO & OBJETIVO & CONCLUSIÓN \\
\hline L 21 & $\begin{array}{l}\text { Prevalence and correlates of } \\
\text { violence against female sex } \\
\text { workers in Abuja, Nigeria } \\
(27) \text {. }\end{array}$ & $\begin{array}{l}\text { Documentar la prevalencia y los tipos de } \\
\text { violencia que sufren las profesionales del } \\
\text { sexo e identificar los factores de riesgo. }\end{array}$ & $\begin{array}{l}\text { La prevalencia de la violencia contra } \\
\text { las trabajadoras del sexo era alta. Es } \\
\text { necesario protegerlas de todas las } \\
\text { formas de violencia. Por lo tanto, es } \\
\text { necesario abordar los desafíos sociales } \\
\text { y económicos que fomentan el trabajo } \\
\text { sexual. }\end{array}$ \\
\hline $\mathrm{L} 22$ & $\begin{array}{l}\text { Scale-up, Retention and } \\
\text { HIV/STI prevalence Trends } \\
\text { among Female sex workers } \\
\text { attending VICITS Clinics in } \\
\text { Guatemala (28). }\end{array}$ & $\begin{array}{l}\text { Presentar la primera evaluación de la } \\
\text { ampliación, la retención, las tendencias } \\
\text { de prevalencia del VIH y las infecciones } \\
\text { de transmisión sexual y los factores } \\
\text { de riesgo asociados a la infección por } \\
\text { el VIH entre las trabajadoras del sexo } \\
\text { que asisten a las clínicas evaluadas en } \\
\text { Guatemala. }\end{array}$ & $\begin{array}{l}\text { Los datos muestran diferencias locales } \\
\text { en la prevalencia del VIH y la atención } \\
\text { clínica que pueden utilizarse para dar } \\
\text { prioridad a las actividades de prevención } \\
\text { para los profesionales del sexo en } \\
\text { Guatemala. Las clínicas han logrado un } \\
\text { rápido aumento de escala; sin embargo, } \\
\text { se necesitan estudios para identificar las } \\
\text { causas de las bajas tasas de rendimiento. }\end{array}$ \\
\hline $\mathrm{L} 23$ & $\begin{array}{l}\text { Tendencias de la prevalencia } \\
\text { del VIH y de las conductas } \\
\text { de riesgo asociadas em } \\
\text { mujeres trabajadoras del } \\
\text { sexo em Cataluña (29). }\end{array}$ & $\begin{array}{l}\text { Describir las tendencias de la prevalencia } \\
\text { del VIH, los comportamientos sexuales } \\
\text { de riesgo y otros tipos de salud sexual en } \\
\text { los indicadores de sexo ocupacional de } \\
\text { las mujeres de } 2005 \text { a } 2011 \text {. }\end{array}$ & $\begin{array}{l}\text { La prevalencia del VIH se ha mantenido } \\
\text { estable a lo largo de los años. El aumento } \\
\text { de las relaciones sexuales sin protección } \\
\text { y la prevalencia de ITS autoreportada } \\
\text { entre el } 2005 \text { y } 2011 \text {, pone de manifiesto } \\
\text { la posible relajación de las conductas } \\
\text { preventivas en este grupo. }\end{array}$ \\
\hline L 24 & $\begin{array}{l}\text { La autoestima como factor } \\
\text { estresor intrapersonal para } \\
\text { el consumo del alcohol em } \\
\text { trabajadoras sexuales }(30) \text {. }\end{array}$ & $\begin{array}{l}\text { Medir la relación entre la autoestima } \\
\text { como factor estresante intrapersonal y el } \\
\text { consumo de alcohol en las trabajadoras } \\
\text { del sexo. }\end{array}$ & $\begin{array}{l}\text { Según el modelo de Betty Neuman } \\
\text { Systems, la baja autoestima tiene un } \\
\text { efecto y un impacto en el consumo de } \\
\text { alcohol. }\end{array}$ \\
\hline
\end{tabular}

\section{DISCUSIÓN}

Sobre la base del análisis de los artículos encontrados, se extrajeron las siguientes categorías: infecciones de transmisión sexual (ITS), violencia y drogas ilegales o lícitas.

Al analizar las publicaciones, se observó que las tres esferas están interconectadas, porque las prostitutas están en una posición altamente vulnerable. Según L1 (7), L2 (8), L6 (12) y L12 (18), la violencia está directamente relacionada con la negociación del uso del preservativo, lo que pone a estas mujeres en mayor riesgo de contraer una ITS. Sin embargo, L1 (7), L11 (17) y L17 (23) también asocian la agresión con el uso de sustancias ilícitas.

Según la L11 (17), el consumo de alcohol antes o durante las relaciones sexuales con los clientes aumentaba la probabilidad de haber sufrido violencia sexual o física alrededor de dos veces. L1 (7) informa que el $52,4 \%$ de las encuestadas declararon haber 
sido amenazadas con tener relaciones sexuales sin preservativo y el 45,6 \% declaró haber sufrido daños físicos.

La violencia fue prevalente entre los artículos seleccionados, tanto de su pareja íntima como de los clientes. Sin embargo, no solo hay agresión física, sino también psicológica, moral y sexual. La violencia contra la mujer incluye la violencia física, que comprende cualquier conducta que ofenda la integridad o la salud corporal de la mujer; la violencia psicológica, que incluye amenazas, humillaciones, aislamiento, persecución, entre otras; la violencia moral, que se caracteriza por la calumnia, la difamación y los insultos; la violencia patrimonial, que se materializa en el robo, la retención o el daño de bienes, recursos y documentos; y la violencia sexual, por relaciones sexuales no deseadas, prácticas sexuales no consentidas y la prevención del uso de anticonceptivos (7 - 13).

Sin embargo, debido a la estigmatización, muchos evitan denunciarla, especialmente cuando la realiza la pareja, por temor a represalias, evitando así mostrar siquiera la prueba del virus de inmunodeficiencia humana/síndrome de inmunodeficiencia adquirida (VIH/SIDA) y la sífilis realizada en la atención prenatal, porque significa infidelidad (31).

La violencia durante el embarazo causa graves daños a la salud de las mujeres, haciendo que algunas desarrollen hipertensión, dolores crónicos, dispareunia, trastornos del sueño, patrones de alimentación y gástricos; además de miedo, depresión, ansiedad, síndrome de estrés postraumático, inseguridad, tristeza y baja autoestima. Aunque el abuso psicológico no deja signos visibles como el abuso físico, es sumamente importante, ya que tiene efectos devastadores tanto para el embarazo como para el puerperio (32 - 34).

Además, como consecuencia física se producen traumatismos abdominales, hemorragias, rotura uterina o rotura prematura de membranas, muerte materna, aborto inducido por agresión y desprendimiento de placenta, favoreciendo la aparición tardía e incluso el abandono del control prenatal por vergüenza o impedimento de la pareja, además del uso de drogas ilegales / lícitas (32 - 34).

Sin embargo, las implicaciones no solo afectan a la madre, sino que el feto puede nacer con bajo peso, presentando restricción del crecimiento, shock fetal y/o materno, prematuridad y muerte fetal o neonatal; es fundamental que la actuación de las enfermeras se base en varias ramas, teniendo en cuenta la situación socioeconómica, familiar y cultural que implica el proceso de atención $(32,34)$.

En lo que respecta a las infecciones de transmisión sexual, los estudios L2 (8), L6 (12), L7 (13), L8 (14), L10 (16), L15 (21), L17 (23), L18 (24) y L23 (29), 24 (30), las asocian con el consumo de drogas y/o las relaciones sexuales sin protección. Según L2 (8), las mujeres, bajo el efecto de las drogas pierden poder de negociación y pueden perder la conciencia de las acciones tomadas $\mathrm{y}$, además, la droga puede presentarse como moneda de cambio para el servicio; además, el uso abusivo de estas sustancias está presente en los acuerdos relativos al uso de preservativos, comprometiendo la decisión como se discute en L6 (12).

Otro factor que comenta L7 (13) es que los propios clientes buscan experiencias de riesgo, porque aumenta la libido durante el acto sexual. Sin embargo, además de los clientes, están las parejas íntimas, que tampoco usan preservativos y pueden adquirir ITS porque son posibles transmisores de estas enfermedades, como se informa en L17 (23) y L18 (24).

Sin embargo, el origen asintomático de algunas y la difícil identificación revelan que muchos casos 
permanecen sin diagnosticar; constituyendo un gran problema para la salud pública, en el que las prostitutas tienen un papel preponderante en la dinámica debido a que la vulnerabilidad de la actividad que realizan las hace susceptible a varias. Entre las infecciones, se encuentran la exposición a la sífilis, el VIH, la gonorrea, la clamidia, el herpes genital, la hepatitis $\mathrm{B}$, la tricomoniasis vaginal, entre otras, y pueden transmitirse a la concepción tanto en el embarazo como en el parto y la lactancia (35).

El VIH y la sífilis son infecciones crónicas con riesgo de transmisión vertical que aparecieron en mayor prevalencia en las publicaciones encontradas, ambas se analizan en las consultas prenatales, sin embargo, por fallas terminan sin diagnosticarse. La tasa de transmisión del VIH en las últimas semanas del embarazo y en la lactancia está entre el $7 \%$ y el $22 \%$, en el parto es del $65 \%$ y en el intrauterino del $35 \%$. La sífilis, en cambio, presenta una tasa de 9,7 casos en mujeres embarazadas por cada 1000 nacidos vivos (36).

En general, los síntomas clínicos de la sífilis congénita son inespecíficos y discretos y pueden conducir a un aborto espontáneo, nacimiento de mortinatos, bajo peso al nacer, prematuridad y complicaciones recientes y tardías en el recién nacido (37). Cuando son recientes, llegan hasta los 2 años de edad, sus características se asemejan a las de la sífilis secundaria, además de la hepatoesplenomegalia, la anemia hemolítica asociada a la ictericia, la periostitis, entre otras. Cuando es tardía, aparece después del $2^{\circ}$ año de vida y las manifestaciones son más raras y pueden involucrar varios órganos, incluyendo la tríada de queratitis intersticial, los dientes de Hutchinson y la sordera del octavo par craneal, además de otras comorbilidades como la nariz en silla de montar, las articulaciones de Clutton y la mandíbula corta (36). Con respecto al VIH, los recién nacidos presentan síntomas en los primeros meses si no han recibido profilaxis; los más comunes son el crecimiento lento y el retraso en la maduración, el aumento de los ganglios linfáticos, la diarrea recurrente, las infecciones pulmonares, la candidiasis, la esplenomegalia, la anemia, la sepsis, las enfermedades cardíacas, la hepatitis, la nefritis y el linfoma no Hodgkin. Sin embargo, si reciben tratamiento los efectos son más leves.

En cuanto al uso de sustancias lícitas e ilícitas, se señaló que están relacionadas con las tasas de violencia y de enfermedades de transmisión sexual en los Servicios Sociales. L15 (21) señala que el consumo de sustancias psicoactivas es un cofactor importante que contribuye al comportamiento sexual, una característica agravante, también citada en el artículo, es que la mayoría de los encuentros se producen en lugares de consumo, favoreciendo el uso. El L7 (13) informa que el alcohol y las drogas ilícitas provocan la disminución y la pérdida del sentido crítico, disminuyendo el cuidado de la salud que comúnmente se toma cuando se está consciente.

Durante el embarazo, el consumo de alcohol es capaz de causar varias consecuencias como: pancreatitis, neuritis, gastritis, hipertonía uterina, riesgo deinfección, nacimiento prematuro, crecimiento intrauterino restringido, prematuridad, presencia de meconio en el líquido amniótico, malformaciones, alteraciones cardíacas y abortos, y el síndrome alcohólico fetal. Además, atraviesa la barrera placentaria exponiendo al feto a la misma concentración absorbida por la madre.

El tabaco es un elemento altamente tóxico que compromete la vitalidad del feto y puede causar patologías hasta la edad adulta, ya que al cruzar la placenta reduce el flujo sanguíneo por vasoconstricción, reduciendo así el oxígeno y los nutrientes al feto, está relacionado con la placenta previa, hemorragias, taquicardia fetal, desprendimiento prematuro de la placenta, abortos y muerte fetal (38).

Cuando se usa concomitantemente con drogas ilícitas 
como el crack, la marihuana y la cocaína, modifica el rendimiento fisiológico de la madre y el bebé con riesgo de retraso en el desarrollo psicomotor y neurocognitivo, reducción de la circunferencia y la estatura craneana, hemorragia intracraneal, malformaciones congénitas, aumento de la posibilidad de nacimientos prematuros, hemorragia anormal, problemas de audición, enfermedades cardíacas y respiratorias, inquietud y síndrome de muerte súbita del lactante (38).

Se pudo observar que las prostitutas están expuestas a varias situaciones y consecuencias durante el período de trabajo, además de poner en riesgo su embarazo y la vida de la concepción, destacando, así, la importancia de los profesionales que trabajan en el área de la salud para reducir los riesgos, principalmente en las consultas prenatales, en lo que respecta al uso de drogas en el embarazo, la violencia y las infecciones de transmisión sexual, para detectarlos precozmente, además de que toda la fase gestacional requiere cuidados específicos.

\section{CONCLUSIÓN}

A pesar del muestreo limitado, el estudio evaluó que el binomio madre-bebé es vulnerable a varias complicaciones como: aborto espontáneo, restricción del crecimiento, parto prematuro, muerte fetal y/o materna, trastornos, prematuridad, anormalidades físicas y de comportamiento, desprendimiento de la placenta debido a la violencia sufrida, infecciones de transmisión sexual adquiridas y sustancias utilizadas. Es importante que la enfermería obstétrica preste atención a esta mujer y la capte en una etapa temprana, alentándola a adherirse más estrechamente a las consultas prenatales para hacerlo de manera correcta y confiable, sin estigmas ni prejuicios. Además, se observó la necesidad de fomentar los estudios que abordan la salud de las trabajadoras del sexo disociándolos del aborto y el abandono.

\section{REFERENCIAS}

1. Salmeron NA, Pessoa TAM. Profissionais do sexo: perfil socioepidemiológico e medidas de redução de danos. Acta Paul Enferm. 2012; 25(4):549-554. doi: http://dx.doi.org/10.1590/S0103-21002012000400011

2. Texeira Rodrigues M. A prostituição no Brasil contemporâneo: um trabalho como outro qualquer? Revis Katál Florianópolis. 2009; 12(1):68-76. doi: http://dx.doi.org/10.1590/S1414-49802009000100009

3. Ministério do Trabalho [Internet]. Brasilia: CBO. Classificação Brasileira de ocupações; 2002 [consultado 15 de marzo de 2019]. Disponible en: http://www. mtecbo.gov.br/cbosite/pages/home.jsf

4. Cavour RC. Mulheres de família: papéis e identidades da prostituta no contexto familiar [tesis en internet]. Rio de Janeiro: Pontifícia Universidade Católica do Rio de Janeiro; 2011 [consultado 15 de marzo de 2019]. Disponible en: https://www.maxwell.vrac.puc-rio.br/ colecao.php?strSecao=resultado\&nrSeq=37393@1

5. Monteiro C, Avelar AFM, Pedreira MLG. Interrupção de atividades de enfermeiros e a segurança do paciente: revisão integrativa da literatura. Rev Latino Am Enfermagem. 2015; 23 (1):169-179. doi: https://doi. org/10.1590/0104-1169.0251.2539

6. Silva T, Bezerra AL, Paranaguá T, Teixeira C. O envolvimento do paciente na segurança do cuidado: revisão integrativa. Rev Eletr Enf. 2016; 18. doi: https://doi.org/10.5216/ree.v18.33340

7. Alemayehu M, Yohannes G, Damte A, Fantahun A, Gebrekirstos K, Tsegay R, et al. Prevalence and predictors of sexual violence among commercial sex workers in Northern Ethiopia. Reprod Health. 2015; 12:47. doi: 10.1186/s12978-015-0036-5. PMID: 26001847; PMCID: PMC4456705

8. Villa EA, Cândido MCRM, Siste LF. A assistência à saúde das profissionais do sexo no Brasil: uma revisão integrativa. J Nurs Health [Internet]. 2016 [consultada 15 de marzo de 2019]; 6(1):92-102. Disponible en: https://docs.bvsalud.org/biblioref/bdenf/2016/bde31720/bde-31720-605.pdf

9. Sousa RM, Frota MM, Castro C, Sousa FB, Kendall BC, Kerr LR. Prostituição, HIV/Aids e vulnerabilidades: a "cama da casa" e a "cama da rua". Cad Saúde Col. 2017; 25(4): 423-428. doi: https://doi.org/10.1590/1414$462 \times 201700040242$

10. Oliveira A, Fernandes L. Trabajadores del sexo y salud pública: intersecciones, vulnerabilidades y resistencia. Salud Colectiva. 2017; 13(2):199-210. doi: http:// dx.doi.org/10.18294/sc.2017.1205 
11. Arboit J, Hirt MC, Gehlen GS, Bortoli VS, Costa MC, Silva EB. Situações de vulnerabilidade à violência de mulheres profissionais do sexo: interfaces no campo da saúde. Rev Enferm UFPE on line. 2014; 8(2):37843789. doi: http://dx.doi.org/10.5205/reuol.4597-376831-ED.0810supl201426

12. Robertson AM, Syvertsen JL, Ulibarri MD, Rangel MG, Martinez G, Strathdee SA. Prevalence and correlates of HIV and sexually transmitted infections among female sex workers and their non-commercial male partners in two Mexico-USA border cities. J Urban Health. 2014; 91(4):752-767. doi: 10.1007/s11524-013-9855-2. PMID: 24488651; PMCID: PMC4134454.

13. Leal CBM, Souza DA, Rios MA. Aspectos de vida e saúde das profissionais do sexo. Rev enferm UFPE on line. 2017; 11(11):4483-4491. doi: http://dx.doi. org/10.5205/reuol.23542-49901-1-ED.1111201726.

14. Nasirian M, Kianersi S, Hoseini SG, Kassaian N, Yaran M, Shoaei P, et al. Prevalence of Sexually Transmitted Infections and Their Risk Factors among Female Sex Workers in Isfahan, Iran: A Cross-Sectional Study. J Int Assoc Provid AIDS Care. 2017; 16(6):608-614. doi: 10.1177/2325957417732836. Epub 2017 Oct 11. PMID: 29017374.

15. Coma Auli N, Mejía-Lancheros C, Berenguera A, Pujol-Ribera E. Risk perception of sexually transmitted infections and HIV in Nigerian commercial sex workers in Barcelona: a qualitative study. BMJ Open. 2015; 5(6):e006928. doi: 10.1136/bmjopen-2014-006928. PMID: 26078307; PMCID: PMC4480001.

16. Chow EP, Fehler G, Chen MY, Bradshaw CS, Denham I, Law MG, et al. Testing commercial sex workers for sexually transmitted infections in Victoria, Australia: an evaluation of the impact of reducing the frequency of testing. PLoS One. 2014; 9(7):e103081. doi: 10.1371/ journal.pone.0103081. PMID: 25048817; PMCID: PMC4105494.

17. Semple SJ, Stockman JK, Pitpitan EV, Strathdee $\mathrm{SA}$, Chavarin CV, Mendoza DV, et al. Prevalence and Correlates of Client-Perpetrated Violence against Female Sex Workers in 13 Mexican Cities. PLoS One. 2015; 10(11):e0143317. doi: 10.1371/ journal.pone.0143317. PMID: 26599083; PMCID: PMC4657898.

18. Cepeda A, Nowotny KM. A border context of violence: Mexican female sex workers on the U.S.-Mexico border. Violence Against Women. 2014; 20(12):150631. doi: 10.1177/1077801214557955. Epub 2014 Nov 18. PMID: 25409891; PMCID: PMC5933926.
19. Awungafac G, Delvaux T, Vuylsteke B. Systematic review of sex work interventions in sub-Saharan Africa: examining combination prevention approaches. Trop Med Int Health. 2017; 22(8):971-993. doi: 10.1111/ tmi.12890. Epub 2017 May 24. PMID: 28449198.

20. Servin AE, Reed E, Brouwer KC, Magis-Rodriguez C, Boyce S, Strathdee SA, et al. Motherhood and Risk for Human Immunodeficiency Virus/Sexually Transmitted Infections Among Female Sex Workers in the MexicoUS Border Region. Sex Transm Dis. 2017; 44(8):477482. doi: 10.1097/OLQ.0000000000000634. PMID: 28703726 ; PMCID: PMC5512441.

21. Longo JD, Simaléko MM, Ngbale R, Grésenguet G, Brücker G, Bélec L. Spectrum of female commercial sex work in Bangui, Central African Republic. SAHARA J. 2017; 14(1):171-184. doi: 10.1080/17290376.2017.1394907. PMID: 29092678; PMCID: PMC5678296.

22. Leite GS, Murray L, Lenz F. The Peer and Non-peer: the potential of risk management for HIV prevention in contexts of prostitution. Rev Bras Epidemiol. 2015; 18 Suppl 1:7-25. English, Portuguese. doi: 10.1590/18094503201500050003. PMID: 26630296.

23. Penha J, Aquino C, Neri É, Reis T, Aquino P, Pinheiro A. Fatores de risco para doenças sexualmente transmissíveis em profissionais do sexo do interior piauiense. Rev Gaúcha Enferm. 2015; 36(2): 63-69. doi: http://dx.doi.org/10.1590/1983-1447.2015.02.52089.

24. Slabbert M, Venter F, Gay C, Roelofsen C, LallaEdward S, Rees H. Sexual and reproductive health outcomes among female sex workers in Johannesburg and Pretoria, South Africa: Recommendations for public health programmes. BMC Public Health. 2017; 17(Suppl 3):442. doi: 10.1186/s12889-017-4346-0. PMID: 28832290; PMCID: PMC5498862.

25. Reis TGO, Penha JC, Neri EAR, Luz GOA, Aquino PS. Health education with prostitutes: an experience to couple of education. Rev Enferm UFPI. 2014; 3(3):4652. doi: https://doi.org/10.26694/reufpi.v3i3.1740.

26. O'Halloran AB, Armstrong G, Medhi GK, Sono CZ, Mahanta J, Kermode M. HIV risks vary according to type of sex work in a cross-sectional survey from Nagaland, India. BMC Womens Health. 2014; 14:133. doi: 10.1186/s12905-014-0133-6. PMID: 25388946; PMCID: PMC4240872.

27. Fawole OI, Dagunduro AT. Prevalence and correlates of violence against female sex workers in Abuja, Nigeria. Afr Health Sci. 2014; 14(2):299-313. doi: 10.4314/ahs. v14i2.4. PMID: 25320578; PMCID: PMC4196411. 
28. Morales-Miranda S, Jacobson JO, Loya-Montiel I, Mendizabal-Burastero R, Galindo-Arandi C, Flores $\mathrm{C}$, et al. Scale-up, retention and HIV/STI prevalence trends among female sex workers attending VICITS clinics in Guatemala. PLoS One. 2014; 9(8):e103455. doi: 10.1371/journal.pone.0103455. PMID: 25167141; PMCID: PMC4148235.

29. Folch C, Casabona J, Sanclemente C, Esteves A, Gonzalez V. Tendencias de la prevalencia del VIH y de las conductas de riesgo associadas en mujeres trabajadoras del sexo en Cataluña. Gac Sanit. 2014; 28(3):196-202. doi: http://dx.doi.org/10.1016/j. gaceta.2013.11.004

30. Salinas Almaguer CE, Padilla N, Ruiz ML, Vera AM. $\mathrm{La}$ autoestima como factor estresor intrapersonal para el consumo de alcohol en trabajadoras sexuales. Enferm Glob [Internet]. 2014 [consultado 15 de marzo de 2019]; 13(33):157-165. Disponible en: http://scielo. isciii.es/scielo.php?script=sci_arttext\&pid $=\mathrm{S} 1695$ 61412014000100007\&lng=es.

31. Teixeira SAM, Taquette SR, Monteiro DLM. Violence and sexually transmitted infections in pregnancy. Rev Assoc Med Bras. 2019; 65(3):475-484. doi: 10.1590/1806-9282.65.3.475. Epub 2019 Apr 11. PMID: 30994850.

32. Netto L, Teizeira S, Moura M, Silva LR, Queiroz ABA, Souza KV. Violência perpetrada por parceiro íntimo à gestante: o ambiente à luz da teoria de Levine. Rev Esc Enferm USP. 2015; 49(6):882-889. doi: https://doi. org/10.1590/S0080-623420150000600002.

33. Ramalho NMG, Ferreira JDL, Lima CLJ, Ferreira T, Umbelino S, Carneiro G. Violência doméstica contra a mulher gestante. Rev Enferm UFPE on line. 2017; 11(12):4999-5008. doi: https://doi.org/10.5205/19818963-v11i12a22279p4999-5008-2017
34. Santos SM, Lovisi GM, Valente CCB, Legay L, Abelha L. Violência doméstica durante a gestação: um estudo descritivo em uma unidade básica de saúde no Rio de Janeiro. Cad Saúde Colet [Internet]. 2010 [consultado 15 de marzo de 2019]; 18(4):483-493. Disponible en: http://www.cadernos.iesc.ufrj.br/cadernos/images/ csc/2010_4/artigos/CSC_v18n4_483-493.pdf

35. Lima SS, Souza LC, Vidal M, Martins JP, Campos M, Espindula M. HIV na gestação: pré-natal, parto e puerpério. Ciênc Saúde. 2017; 10(1):56-61. doi: http:// dx.doi.org/10.15448/1983-652X.2017.1.22695.

36. Costa CV, dos Santos IA, da Silva JM, Fernandez T, Silva H. Sífilis congênita: repercussões e desafios. Arq Catarin Med [Internet]. 2017 [consultado 15 de marzo de 2019]; 46(3):194-202. Disponible en: http:// www.acm.org.br/acm/seer/index.php/arquivos/article/ view/94

37. Horta HL, Martin MF, Nonato TF, Alves MI. Pré-natal do parceiro na prevenção da sífilis congênita. APS. 2017; 20(4):623-627.doi: https://doi.org/10.34019/18098363.2017.v20.16078

38. Teles da Reis Rocha R. Drogas na gravidez e consequências em recém-nascidos. J Spec [Internet]. 2018 [consultado el 15 de marzo de 2019]; 1(2). Disponible en: http://www.journalofspecialist.com.br/ jos/index.php/jos/article/view/81

Recibido 9 de junio de 2020 Aprobado 30 de septiembre de 2020 